\title{
QSAR and its Role in Target-Ligand Interaction
}

\author{
Dr. Anamika Singh ${ }^{1, *}$ and Dr Rajeev Singh ${ }^{2, *}$ \\ ${ }^{1}$ Maitreyi Collage, University of Delhi, India \\ ${ }^{2}$ Indian Council of Medical Research, New Delhi, India
}

\begin{abstract}
Each molecule has its own specialty, structure and function and when these molecules are combined together they form a compound. Structure and function of a molecule are related to each other and QSARs (Quantitative StructureActivity relationships) are based on the criteria that the structure of a molecule must contain the features responsible for its physical, chemical, and biological properties, and on the ability to represent the chemical by one, or more, numerical descriptor(s). By QSAR models, the biological activity of a new or untested chemical can be inferred from the molecular structure of similar compounds whose activities have already been assessed. QSARs attempt to relate physical and chemical properties of molecules to their biological activities. For this there are so many descriptors (for example, molecular weight, number of rotatable bonds, Log P) and simple statistical methods such as Multiple Linear Regression (MLR) are used to predict a model. These models describe the activity of the data set and can predict activities for further sets of (untested) compounds. These types of descriptors are simple to calculate and allow for a relatively fast analysis. 3D-QSAR uses probe-based sampling within a molecular lattice to determine three-dimensional properties of molecules (particularly steric and electrostatic values) and can then correlate these 3D descriptors with biological activity. Physicochemical descriptors, include hydrophobicity, topology, electronic properties, and steric effects etc. These descriptors can be calculated empirically, statistically or through more recent computational methods. QSARs are currently being applied in many disciplines, with many pertaining to drug design and environmental risk assessment.
\end{abstract}

Key word: QSAR, Ligand Designing, LogP, Cheminformatics, Docking.

\section{INTRODUCTION}

Quantitative structure-activity relationship (QSAR) has been playing a major role in the field of agriculture chemistry, pharmacology, and toxicology since last few years [1]. With the help of QSAR, we can design new models and compare them with existing models or newly generated models to the biological databases. On the basis of similarity and dissimilarity, we can also conclude the relationship between these two. Quantitative structureactivity relationship (QSAR) modeling is an area of research which was pioneered by Hansch and Fujita $[2,3]$. The QSAR study assumes that the difference of the molecules in the structural properties experimentally measured accounts for the difference in their observed biological or chemical properties [4]. With the help of QSAR, it is now possible not only to develop a model for a system but also to compare models from a biological database and to draw analogies with models from a physical organic database [5]. QSAR is able to identify the relationship between a molecule and its structure and how the structure influences the activity of the molecule. There are few parameters like steric properties, electron distribution and hydrophobicity etc. In spite of this, minute analysis shows that there are some small parameters

*Address correspondence to this author at the Indian Council of Medical Research, New Delhi, India; Tel: 09810238056, 08826125568;

E-mails: rajeev_mbt@rediffmail.com 10rsingh@gmail.com which can affect the function of a molecule and are generally known as molecular descriptors. These descriptors are atomic descriptors and are derived from quantum chemical calculations and spectroscopy. [6]. High-throughput screening method allows fast screening of large number of dataset. It separates the molecule with similar structure and function. This method is very useful as it helps to minimize the risk of comparison between different dataset of variable sources. From a drug development to its mode of action all steps can be compared easily along with drug formulation by using this method. QSAR method does not only compare the dataset but also generates the data of their analogy [7]. More recently, QSAR has been extended by inclusion of 3D information. In drug discovery, it is common to have measured activity data for a set of compounds acting upon a particular protein, but not to have knowledge of the three dimensional (3D) structure of the active site. In the absence of such 3D information, one may attempt to build a hypothetical model of the active site that can provide insight into the nature of the latter. Three-dimensional approaches such as HypoGen and/or Hip Hop are useful in building 3D pharmacophore models from the activity data and conformational structure [8]. In spite of this, a new method of QSAR having a multiple field three-dimensional quantitative structure-activity relationship (MF-3D-QSAR), is proposed. It is a combination and advanced form of classical 2D-QSAR and traditional 3D-QSAR. In addition to the electrostatic and van der Waals potentials, more potential fields (such as lipophilic potential, hydrogen bonding 
potential, and non-thermodynamic factors) are integrated in the MF-3D-QSAR [9].

Highly predictive QSAR and CoMFA models are being developed using the technique of structure-based alignments. These models explain the protein-ligand interactions and are found consistent with the crystal structure of protein with ligand. CoMFA (Comparative Molecular Field Analysis) has become one of the most powerful tools for QSAR and drug design. In fact, CoMFA has been pioneered as a new paradigm of three-dimensional QSAR where the shapes, properties, etc. of molecules are related to specific molecular features (substituents etc.) and their spatial relationship. The qualitative information of CoMFA contour maps gives key features on not only the ligand-receptor interaction but also on the topology of the receptor. However comparative molecular field analysis (CoMFA) is critically dependent on the conformation and alignment criteria for molecules, and thus among the plethora of methods available for superimposition, the pharmacophoric alignments are considered to be the best [10-16]. Three-dimensional quantitative structure-activity relationship (3D-QSAR) programs such as the Hypo Gen module of Catalyst $[17,18]$, Phase [19], CoMFA [20] and CoMSIA [21] are available. These tools predict the three-dimensional alignments of molecules to quantitatively predict the activity of compounds, on the basis of QSAR models fitted to the activity of an aligned training set of molecules. Such 3D models are more easily interpretable than 2D descriptor or fingerprint-based QSAR models. It should also be possible to make connections from such activity models to structurebased design, either to add more information to overlays for the construction of a pharmacophore model [22, 23]. Quantitative structure-activity relationship (QSAR) represents an approach to correlate structural descriptors of compounds with their biological activities. Threedimensional quantitative structure activity-relationship (3DQSAR) studies provide deeper insight into the mechanism of action of compounds that ultimately becomes of great importance in modification of the structure of compounds. In addition, 3D-QSAR also provides quantitative models, which permits prediction of activity of compounds prior to the synthesis. Self organizing molecular field analysis (SOMFA) is a novel 3D-QSAR methodology which has been developed by Robinson et al. [24].

A pharmacophoric hypothesis collects common features distributed in three-dimensional space representing groups in a molecule that participate in important interactions between drugs and their active sites. Hence a pharmacophore model provides crucial information about how well the common features of a subject molecule overlap with the hypothesis model. In addition, it also helps in the identification of the energetically reasonable conformation, which fits to the active site. Such characterized three-dimensional models convey important information in an intuitive manner. In addition to the standard steric and electronic field used in CoMFA, several types of fields have been evidenced in advanced CoMFA. These are, the hydrogen bonding fields, which are created by assigning energies equal to the steric cutoff energy to lattice points that are close to H-bond accepting or donating atoms, and the indicator fields, which are created by squaring the original field at each lattice point, with the retention of the sign of the original field. Also as an alternative approach to the computation of molecular potential, the comparative molecular similarity indices analysis (CoMSIA) has been developed [25]. It is a simple and intuitive in concept and avoids the complex statistical tools and variable selection procedures favored by other methods. The method has similarities to both comparative molecular field analysis (CoMFA) and molecular similarity studies [26]. Like CoMFA, a grid-based approach is used; however, no probe interaction energies need to be calculated. Like the similarity methods, it has the intrinsic molecular properties, such as the molecular shape and electrostatic potential, which are used to develop QSAR models [27]. A SOMFA model could be based on any molecular property. In the present study, we have used molecular shape and electrostatic potentials. A successful 3D-QSAR model does not only help in better understanding of the structure-activity relationship of any class of compounds, but also provides researcher an insight at molecular level about lead compounds for further developments. The inherent simplicity of this method allows the possibility of aligning the training compounds as an integral part of the model derivation process and of aligning prediction compounds to optimize their predicted activities [28]. Recent studies in our laboratory have focused on refining the molecular architecture using 3D-QSAR SOMFA approach for designing and optimization of new inhibitors for various targets $[29,30]$.

Latest technologies of QSAR involve three different aspects.

(1) Fragment based 2D QSAR (FB 2D-QSAR): In this method, a drug candidate is first subjected to fragmentation into its components and then bioactivities of drug candidates are correlated with physicochemical properties of the molecular fragments through two sets of coefficients: one is for the physicochemical properties and the other for the molecular fragments [31]. FB-QSAR is used to predict the model of neuraminidase inhibitors for drug development against H5N1 influenza virus [32]. This method is also used to predict the new insights for dealing with the drug-resistant problem and designing effective adamantane-based anti-flue drugs for influenza A viruses [33].

(2) Multiple field 3D QSAR (MF 3D QSAR): It is based on comparative molecular field analysis (CoMFA) in which two sets of coefficients are involved: one is for the potential fields and the other for the Cartesian three dimensional grid points [31].

(3) Amino Acid-Based Peptide Prediction (AABPP): In this method, the biological activities of the peptides and proteins are correlated with the physicochemical properties of all or partial residues of the sequence through two sets of coefficients: one is for the physicochemical properties of amino acids and the other for the weight factors of the residues [31].

There is a clear cut limitation of QSAR as it can be onetask or one-target Quantitative Structure-Activity Relationships (ot-QSAR) that predict the biological activity of drugs against only one parasite species. Consequently, multi-tasking learning used to predict drugs activity against different species by a single model (mt-QSAR) becomes vitally important. The mt-QSARs offer a good opportunity 
(unpractical with ot-QSAR) to construct drug-drug similarity Complex Networks and to map the contribution of sub-structures to function for multiple species. Prado et al., in 2009 first tried to calculate probabilities of antiparasitic action of drugs against different parasites [34]. These mtQSARs offer a good opportunity to construct drug-drug Complex Networks (CNs) that can be used to explore large and complex drug-viral species databases. mt-QSAR models are used to predict the antimicrobial activity against different fungi [35], bacteria [36] or parasite species [37, 38].

\section{APPLICATIONS OF QSAR}

A normal QSAR analysis leads to formation of so many equations as it compares and generates a large number of dataset for biological systems. Hansch was the first to give an equation for drug-receptor interactions, in which he considered that electronic, steric, and hydrophobic contributions are the major factors which affect the interaction. Utilizing a nonlinear relationship between them and through pharmaco-kinetics, the drug-receptor interaction can be tracked at cellular level. Kubinyi proposed a bilinear model and supported the concept of delineation of drugreceptor interaction. Dihydrofolate reductases (DHFRs) and a-chymotrypsin interaction was studied in detail.

\section{Isolated Receptor Interactions}

Dihydrofolate reductases (DHFRs) play an important role in protein, purine, and pyrimidine synthesis. They have a crystallographic structure and are able to form a binary and ternary complex. These specialties make the DHFRs as a successful target for some heterocyclic ligand like 2, 4diamino-1,-3-diazapharmacophore [39].

\section{Interactions at the Cellular Level}

QSAR analysis shows all the physico-chemical parameters involved in the studies at the cellular level and at it also tracks the pharmacokinetics. Cell culture method can also be performed to support the QSAR studies. Extensive QSAR studies have proved that 3-X-triazines possess toxic effect on few mammalian and bacterial cell lines [40, 41]. Cytotoxicity levels have been compared between murine leukemia cells (L1210/S) and methotrexate-resistant murine leukemia cells (L1210/R) and this comparison shows some difference among the both.

\section{In Vivo Interactions}

QSAR helps to compare a large dataset with in a limited time and may also lead to heterogeneity and complexity of the data. Only few studies of QSAR have been carried out by generating a very small number of datasets [42]. The best example is an extensive study between renal and no renal clearance rates among few blockers. These blockers are eleven in numbers namely bufuralol, tolamolol, propranolol, alprenolol, oxprenolol, acebutol, timolol, metoprolol, prindolol, atenolol, and nadolol. All of these were compared and studied by QSAR [43].

\section{Three Dimensional Pharmacophore Modeling of Human CYP17 Inhibitors}

Using the pharmacophore perception technique, a commonfeature pharmacophore model(s) was generated to explain the putative binding requirements for two classes of human CYP17 inhibitors (Potential Agents for Prostate Cancer Therapy). Common chemical features in the steroid and nonsteroid human CYP17 enzyme inhibitors, as deduced by the Catalyst/HipHop program, are one to two hydrogen bond acceptors (HBAs) and three hydrophobic groups. For azolesteroidal ligands, the 3 '-OH group of ring $\mathrm{A}$ and the $\mathrm{N}-3$ group of the azole ring attached to ring $\mathrm{D}$ at $\mathrm{C}-17$ act as hydrogen bond acceptors. A model that permits hydrogen bond interaction between the azole functionality on ring $\mathrm{D}$ and the enzyme is consistent with experimental deductions for type II CYP17 inhibitors where a sixth ligating atom interacts with $\mathrm{Fe}(\mathrm{II})$ of heme. In general, pharmacophore models derived for steroid and nonsteroidal compounds bear striking similarities to all azole sites mapping the HBA functionality and to three hydrophobic features describing the hydrophobic interactions between the ligands and the enzyme. Using the pharmacophore model derived for azolesteroidal inhibitors as a 3D search query against several 3D multi conformational Catalyst formatted databases, we identified several steroidal compounds with potential inhibition of this enzyme. Biological testing of some of these compounds showed low to high inhibitory potency against the human CYP17 enzyme. This shows the potential of our pharmacophore model in identifying new and potent CYP17 inhibitors. Further refinement of the model is in progress with a view to identify and optimize new leads [44].

\section{Potential Agents for Prostate Cancer Therapy}

From studies it has been identified in detail, the structural elements of naphthoquinone derivatives that are necessary for cytotoxic activity in the HL-60 cell line. The independent generation of a HypoGen pharmacophore model and a QSAR/CoMSIA model, using the alignment obtained with the former, has been shown to be a valuable tool for analysis. Highly active compounds, such as beta-cycled pyran-1, 2naphthoquinones $[0.1 i \mathrm{M}<\mathrm{IC} 50<0.57 i \mathrm{M}]$, contain four pharmacophoric features: three hydrogen-bond-acceptor groups and a hydrophobic region. These results provide the tools for the design and synthesis of new ligands with high predetermined activities.

Catalyst/HypoGen pharmacophore modeling approach and three-dimensional quantitative structure-activity relationship (3D-QSAR)/comparative molecular similarity indices analysis (CoMSIA) methods have been successfully applied to explain the cytotoxic activity of a set of 51 natural and synthesized naphthoquinone derivatives tested in human promyelocytic leukemia HL-60 cell line. The computational models have facilitated the identification of structural elements of the ligands that are essential for antitumoral properties. The four most salient features of the highly active $\hat{a}$-cycled-pyran-1, 2-naphthoquinones $[0.1 \quad i \mathrm{M}<\mathrm{IC} 50<0.6$ $i \mathrm{M}$ ] are the hydrogen-bond interactions of the carbonyl groups at C-1 (HBA1) and C-2 (HBA2), the hydrogen bond interaction of the oxygen atom of the pyran ring (HBA3), 
and the interaction of methyl groups (HYD) at the pyran ring with a hydrophobic area at the receptor. The moderately active 1, 4-naphthoquinone derivatives accurately fulfill only three of these features. The results of our study provide a valuable tool in designing new and more potent cytotoxic analogs [45].

\section{QSAR and Pharmacophore Analysis on Amides Against Drug-Resistant}

A 3D pharmacophore model able to quantitatively predict inhibition constants was derived for a series of inhibitors of Plasmodium falciparum dihydrofolate reductase (PfDHFR), a validated target for anti-malarial therapy. The data set included 52 inhibitors, with 23 of these comprising the training set and 29 an external test set. The activity range, expressed as $\mathrm{Ki}$, of the training set molecules was from 0.3 to $11300 \mathrm{nM}$. The 3D pharmacophore, generated with the HypoGen module of Catalyst 4.7, consisted of two hydrogen bond donors, one positive ionizable feature, one hydrophobic aliphatic feature, and one hydrophobic aromatic feature and provided a 3DQSAR model with a correlation coefficient of 0.954 . Importantly, the type and spatial location of the chemical features encoded in the pharmacophore were in full agreement with the key binding interactions of PfDHFR inhibitors as previously established by molecular modeling and crystallography of enzyme-inhibitor complexes. The model was validated using several techniques, namely, Fisher's randomization test using Cat-Scramble, leave-oneout test to ensure that the QSAR model is not strictly dependent on one particular compound of the training set, and activity prediction in an external test set of compounds. In addition, the pharmacophore was able to correctly classify as active and inactive dihydrofolate reductase and aldose reductase inhibitors extracted from the MDDR database, respectively. This test was performed in order to challenge the predictive ability of the pharmacophore with two classes of inhibitors that target very different binding sites. Molecular diversity of the data sets was finally estimated by means of the Tanimoto approach. The results obtained provide confidence for the utility of the pharmacophore in the virtual screening of libraries and databases of compounds to discover novel PfDHFR inhibitors [46].

\section{QSAR Studies for Dynein, Pyrazole and their Derivatives}

Dea-Ayuela et al. have found new drug targets for the two species of Leshmania, Leishmania infantum and Leishmania major. Dyneins are important proteins and perform major function in the vital activities, such as motion of cilia and flagella, nuclear migration, organization of the mitotic spindle, and separation of chromosome during mitosis. Dea-Ayuela et al. have successfully identified $L$. infantum protein containing dynein heavy chain, and illustrated the potential use of the QSAR model as a complement to alignment tools [47]. Similarly quantum chemical and structure-based technique known as heuristic molecular lipophilicity potential (HMLP) method is used for alcohol dehydrogenase (LADH) studies of molecular family pyrazole and derivatives. The molecular lipophilic index LM, molecular hydrophilic index HM, lipophilic indices and hydrophilic indices of the substitutes (fragments) and atomic lipophilicity indices are constructed and used in QSAR study. HMLP indices are correlated with bioactivities of 18 pyrazole derivatives according to the $2 \mathrm{D}$ QSAR procedure [48].

\section{CONFLICT OF INTEREST}

The authors confirm that this article content has no conflicts of interest.

\section{ACKNOWLEDGEMENT}

Declared none.

\section{REFERNECES}

[1] C. Hansch, and A. Leo, Substituent constants for correlation analysis in chemistry and biology. John Wiley \& Sons: New York, 1979.

[2] C. Hansch, and A. Leo, In Exploring QSAR: Fundamentals and applications in chemistry and biochemisry. American Chemical Society: Washington DC, 1995.

[3] C. Hansch, and C. Selassie, Quantitative structure-activity relationship-a historical perspective and the future. Elsevier; Oxford, 2007.

[4] V. E. Golender and E. R. Vorpagel, In 3D-QSAR in drug design: Theory, methods, and application. Kubinyi, H., Ed.; ESCOM Science Publishers: The Netherlands, 1993, p. 137.

[5] C. Hansch, A. Kurup, R. Garg, and H. Gao, "Chem-bioinformatics and QSAR: a review of QSAR lacking positive hydrophobic terms", Chem. Rev., vol. 101, p. 619, 2001.

[6] D. J. Livingstone, "The characterization of chemical structures using molecular properties. A survey", J. Chem. Info. Comput. Sci., vol. 40, pp. 195-209, 2000.

[7] U. B. Pandey, and C. D. Nichols, "Human disease models in Drosophila melanogaster and the role of the fly in therapeutic drug discovery", Pharmacol. Rev., vol. 63, pp. 2411-436, 2011.

[8] E.M. Krovat, K.H. Fruhwirth, and T. Langer, "Pharmacophore Identification, in silico screening, and virtual library design for inhibitors of the human factor Xa", J. Chem. Inf. Model., vol. 45, pp. 146-159. 2005.

[9] Q. S. Du, R. B. Huang, Y. T. Wei, "Multiple field three dimensional quantitative structure-activity relationship (MF-3DQSAR)", J. Comput. Chem., vol. 29, pp. 211-219, 2008.

[10] U. Norinder, "The alignment problem in 3D-QSAR: A combined approach using catalyst and a 3D-QSAR technique", J. Chem. Inf. Model., vol. 45, 2005.

[11] U. Norinder,. F. Sanz, J. Giraldo, and F. Manaut, Eds., The Alignment problem in 3D-QSAR: A combined approach using catalyst and a 3D-QSAR technique, In: QSAR and molecular modeling: concepts, computational tools and biological applications. Prous Science Publishers: Barcelona, pp. 433-438, 1995.

[12] R. Hoffmann, T. Langer, T. F. Sanz, J. Giraldo, F. Manaut, Eds., Use of the catalyst program as a new alignment tool for $3 D-Q S A R$. QSAR and molecular modelling: Concepts, computational tools and biological applications. Prous Science Publishers: Barcelona, pp. 466-469, 1995.

[13] S. F. Semus, "CoMFA: A field of dreams?" http://www.netsci.org/ Science/Compchem/feature11.html

[14] T. Langer, and Hoffmann, R. D, "On the use of chemical functionbased alignments as input for 3D-QSAR". J. Chem. Info. Comput. Sci., vol. 38, pp. 325-330, 1998.

[15] R. Bureau, C. Daveu, I. Baglin, J. S. O. Santos, J.C. Lancelot, and S. Rault, "Association of two 3D QSAR analyses. Application to the study of partial agonist serotonin-3 ligands", J. Chem. Info. Comput. Sci., vol. 41, pp. 815-823, 2001.

[16] L. L. Zhu, T.J. Hou, L.R. Chen, and X.J. Xu, “3D QSAR analyses of novel tyrosine kinase inhibitors based on pharmacophore alignment". J. Chem. Info. Comput. Sci., vol. 41, pp. 1032-1040, 2001 . 
[17] Y. Kurogi, and O.F. Guner, "Pharmacophore modelling and threedimensional database searching for drug design using catalyst", Curr. Med. Chem., vol. 8, pp. 1035-1055, 2001.

[18] H. Li, J. Sutter, R. Hoffman, hypogen: an automated system for generating $3 \mathrm{~d}$ predictive pharmacophore models. In: O. Guner, Ed., Pharmacophore perception, development and use in drug design. International University Line: La Jolla, CA, 2000.

[19] Dixon, S. et al., "PHASE: A new engine for pharmacophore perception, 3D QSAR model development, and 3d database screening: 1. Methodology and preliminary results", J. Comput. Aided Mol. Des., vol. 20, pp. 647-671, 2006.

[20] R.D. Cramer, D. Patterson, J. Bunce, "Comparative molecular field analysis (CoMFA). 1. Effect of shape on binding of steroids to carrier proteins", J. Am. Chem. Soc., vol. 110, pp. 5959-5967, 1988.

[21] G. Klebe, U. Abraham, T. Mietzner, "Molecular similarity indices in a comparative analysis (CoMSIA) of drug molecules to correlate and predict their biological activity", J. Med. Chem., vol. 37, pp. 4130-4134, 1994

[22] A. Evers, H. Gohlke, and G. Klebe, "Ligand-supported homology modelling of protein binding-sites using knowledge-based potentials", J. Mol. Biol., vol. 334, pp. 327-345, 2003.

[23] A. Tafi, C. Bernardini, M. Botta, F. Corelli, M. Andreini, A. Martinelli, G. Ortore, P.G. Baraldi, F. Fruttarolo, P.A. Borea, T. Tuccinardi, "Pharmacophore based receptor modeling: the case of adenosine a3 receptor antagonists". J. Med. Chem., vol. 49, pp. 4085-4097, 2006.

[24] D. D. Robinson, P. J. Winn, P.D. Lyne, W. G. Richards, "Selforganizing molecular field analysis: A tool for structure-activity studies. J. Med. Chem., vol. 42, pp. 573-583, 1999.

[25] G. Klebe, U. Abraham, T. Mietzner, "Molecular similarity indices in a comparative analysis (comsia) of drug molecules to correlate and predict their biological activity", J. Med. Chem., vol. 37, pp. 4130-4146, 1994.

[26] R. D. Cramer, D. E. Patteerson, and J. D. Bunce, "Comparative molecular field analysis (CoMFA). 1. Effect of shape on binding of steroids to carrier proteins", J. Am. Chem. Soc., vol. 110, pp. 59595967, 1988.

[27] L. Li, and Y. Zheng, "Self-organizing molecular field analysis on a new series of $\mathrm{COX}-2$ selective inhibitors: 1,5diarylimidazoles". Int. J. Mol. Sci., vol. 7, pp. 220-229, 2006.

[28] M. Y. Li, L.P. Du, B. Wu, and L. Xia, "Self-organizing molecular field analysis on $\alpha_{1 a^{-}}$adrenoceptor dihydropyridine antagonists", Bioorg. Med. Chem., vol. 11, 3945-3951, 2003

[29] S. Thareja, S. Aggarwal, and T. R. Bhardwaj, M. Kumar, "Selforganizing molecular field analysis of 2,4-thiazolidinediones: A 3D-QSAR model for the development of human PTP1B inhibitors", Eur. J. Medi. Chem., vol. 44, pp. 4920-4925, 2009.

[30] S. Aggarwal, S. Thareja, T. R. Bhardwaj, M. Kumar, "3D-QSAR studies on unsaturated 4-azasteroids as human 5alpha-reductase inhibitors: a self organizing molecular field analysis approach", Eur. J. Medi. Chem., vol. 45, pp. 476-481, 2010.

[31] Q.S. Du, R.B. Huang, K.C. Chou, "Review: Recent advances in QSAR and their applications in predicting the activities of chemical molecules, peptides and proteins for drug design", Curr Prot Pept Sci., vol. 9, pp. 248-259. 2008.

[32] Q.S. Du, R.B. Huang, Y. T. Wei, Z.W. Pang, and L.Q. Du, "Fragment-Based quantitative structure-activity relationship (FBQSAR) for fragment-based drug design", J. Comput. Chem., vol. 30, pp. 295-304, 2009

[33] H. Wei, C. H. Wang, Q. S. Du, J. Meng, "Investigation into adamantane-based M2 inhibitors with FB-QSAR". Med. Chem., vol. 5, pp. 305-317, 2009.

[34] F. J. Prado-Prado, H. Gonzalez-Diaz, O. M. de la Vega, "Unified QSAR approach to antimicrobials. Part 3: First multi-tasking
QSAR model for Input-Coded prediction, structural backprojection, and complex networks clustering of antiprotozoal compounds", Bioorg. Med. Chem., vol. 16, pp. 5871-5880, 2008.

[35] H. Gonzalez-Diaz, F. J. Prado-Prado, L. Santana, E. Uriarte, "Activity of Lupane triterpenoids from Maytenus species as nitic oxide and Prostaglandin E2s", Bioorg. Med. Chem., vol. 14, pp. 5973-1579, 2006.

[36] F. J. Prado-Prado, H. Gonzalez-Diaz, L. Santana, E. Uriarte, "Unified approach of QSAR to antimicrobial Part 2: predicting activity against more then 90 different species in order to halt antibacterial resistant", UniBioorg. Med. Chem., vol. 15, pp. $897-$ 913, 2007.

[37] F. J. Prado-Prado, H. González-Díaz, O. Martinez de la Vega, F. M. Ubeira, K. C. Chou, "Unified QSAR approach to antimicrobials. Part 3: first multi-tasking QSAR model for inputcoded prediction, structural back-projection, and complex networks clustering of antiprotozoal compounds", Bioorg. Med. Chem., vol. 16 , p. 5871,2008

[38] F. J. Prado-Prado, O. Martinez de la Vega, E. Uriarte, "Unified QSAR approach to antimicrobials. 4. Multi-target QSAR modeling and comparative multi-distance study of the giant components of antiviral drug-drug complex networks", Bioorg, Med. Chem., vol. 17, pp. 569-575, 2009.

[39] J. M. Blaney, C. Hansch, C. Silipo, A. Vittoria, "Structure-activity relationships of dihydrofolate reductase inhibitors", Chem. Rev., vol. 84: pp. 333-407, 1984.

[40] J. Devillers, Eds. Comparative QSAR. Taylor \& Francis: Washington, DC, 1998.

[41] D. Selassie, C. D. Strong, C. Hansch, T. J. Delcamp, J. H Freisheim, and T. A. Khwaja, "Comparison of triazines as inhibitors of L1210 dihydrofolate reductase and of L1210 cells sensitive and resistant to methotrexate". Cancer Res., vol. 46, pp. 744-756, 1986

[42] Hansch, A. Leo, and D. Hoekman, Eds., Exploring QSAR: Hydrophobic, electronic, and steric constants. ACS: Washington, 1995.

[43] P. H. Hinderling, O. Schmidlin, and J. K. Seydel, "Quantitative relationships between structure and pharmacokinetics of betaadrenoceptor blocking agents in man",J. Pharmacokinet. Biopharm., vol. 12, no. 3, pp. 263-287, 1984.

[44] O. O. Clement, C. M. Freeman, R. W. Hartmann, V. D. Handratta, T. S. Vasaitis, A. M. Brodie and V. C. Njar, "Three dimensional pharmacophore modeling of human CYP17 inhibitors. Potential agents for prostate cancer therapy", J. Med. Chem., vol. 46, pp. 2345-2351, 2003.

[45] Pe'rez-Sacau, R. G. D1'az-Peñate, A. Este'vez-Braun, A.G. Ravelo, J. M. Garcı'a-Castellano, L. Pardo, and M. Campillo, "Synthesis and pharmacophore modeling of naphthoquinone derivatives with cytotoxic activity in human promyelocytic leukemia hl-60 cell line", J. Med. Chem., vol. 50(4), pp.696-706, 2007 .

[46] Parenti, S.Pacchioni, A.M. Ferrari, and G. Rastelli, "ThreeDimensional quantitative structure-activity relationship analysis of a set of plasmodium falciparum dihydrofolate reductase inhibitors using a pharmacophore generation approach", Marco. J. Med. Chem., vol. 47, pp. 4258-4267, 2004.

[47] M. A. Dea-Ayuela, Y. Perez-Castillo, A. Meneses-Marcel, F. M Ubeira, and F. Bolas-Fernandez, "HP-Lattice qsar for dynein proteins: experimental proteomics (2d-electrophoresis, mass spectrometry) and theoretic study of a leishmania infantum sequence", Bioorg. Med. Chem., vol. 16, pp. 7770-7776, 2008.

[48] Q. S. Du, and P. G. Mezey, "Heuristic molecular lipophilicity potential (hmlp): a 2d-qsar study to ladh of molecular family pyrazole and derivatives, J. Comput. Chem., vol. 26, pp. 461-470, 2005. 